\title{
Effect of Danggui Sini Decoction on the Behaviour and Dorsal Root Ganglion TRP Channel of Neuropathic Pain in CCI Rats
}

\author{
D. ZHANG, D. CHEN¹, SHENGSUO MA, WENYAN BIAN, F. XIE, MEINA HUANG, HAOMING LIN, QIANXING ZHAN AND \\ G. ZHAO* \\ College of Traditional Chinese Medicine, Jinan University, Guangzhou, 510630, ${ }^{1}$ LiWan Hospital of Traditional Chinese Medicine, \\ 142 GuangYa Road, Guangzhou, Guangdong 510665, China
}

Zhang et al.: Effect of Danggui Sini Decoction for neuropathic pain in CCI rats

\begin{abstract}
Danggui Sini decoction, a traditional Chinese medicine formula, has been extensively used as a remedy for neuropathic pain and other diseases in recent years. To explore whether Danggui Sini decoction can alleviate mechanical pain, thermal hyperalgesia, and cold pain at the same time, the role of temperaturesensitive transient receptor potential channel in neuropathic pain and the therapeutic effect of Danggui Sini decoction on neuropathic pain as well as the impact on the transient receptor potential pathway were explored in the present study. One hundred and twenty-eight Sprague Dawley rats were separated randomly into 4 groups; the sham-operated group, chronic constriction injury of sciatic nerve model group, pregabalin group, and Danggui Sini decoction-treated group. The rats in the sham-operated group underwent surgery, but no nerve was ligated. The rest of the rats were subjected to sciatic nerve ligation. Rats in Danggui Sini decoction-treated group could be irrigated to Danggui Sini decoction. Rats in the sham-operated group and chronic constriction injury model group were gavaged with saline. Rats in pregabalin group were given pregabalin. Behavioural tests conducted were, acetone test and cold plate, hot plate experiment, and von Frey filaments on 4, 7, 14, 21 st day, and then dorsal root ganglion neurons were taken out. Nerve ligation from the ipsilateral L4-6 dorsal root ganglion was measured TRPM8, TRPA1, TRPV1, TRPV2, TRPV3, and TRPV4 proteins expression by immunofluorescence staining and Western-blot method. Danggui Sini decoction could significantly inhibit the cold allodynia and the mechanical allodynia. The immunofluorescence studies showed that the model group of dorsal root ganglia on TRPM8, TRPA1, TRPV1, TRPV2, TRPV3, and TRPV4 proteins were decreased. Western-blot results showed the expression of TRPM8, TRPA1, TRPV1, TRPV2, TRPV3, and TRPV4 proteins in Danggui Sini decoction-treated group was reduced. Danggui Sini decoction could not only suppress the cold allodynia, but also alleviate mechanical allodynia and thermal hyperalgesia. Danggui Sini decoction could down-regulate abnormally high expression of temperaturedependent transient receptor potential channel proteins.
\end{abstract}

Key words: Transient receptor potential channel, Danggui Sini decoction, dorsal root ganglion, neuropathic pain

Neuropathic pain (NP), a highly complex and prevalent disease, is common clinically but without effective treatments ${ }^{[1]}$. It can cause substantial disability in patients, reduce quality of life, and potentially result in a chain of secondary complications. NP is supposed to be characterized by spontaneous pain, hyperalgesia, an enhanced pain evoked by a noxious stimulus, and allodynia, pain evoked by innocuous stimulus ${ }^{[2]}$. In fact, a variety of diseases, trauma, infection, surgery, tumour, metabolic diseases, chemoradiotherapy, can give rise to NP. NP is an intricate phenomenon, including various ascending and descending neuronal

*Address for correspondence E-mail: tguo428@jnu.edu.cn

September-October 2019 pathways, and involving the central nervous system ${ }^{[3]}$. The mechanism is unclear, but recent reports have indicated that peripheral sensitization and central sensitization are correlated with $\mathrm{NP}^{[4,5]}$. The peripheral sensitization can cause central sensitization; and

This is an open access article distributed under the terms of the Creative Commons Attribution-NonCommercial-ShareAlike 3.0 License, which allows others to remix, tweak, and build upon the work non-commercially, as long as the author is credited and the new creations are licensed under the identical terms

Accepted 16 August 2019

Revised 22 May 2019

Received 14 Janaury 2019

Indian J Pharm Sci 2019;81(5):922-932 
after central sensitization, it does not depend on peripheral injury stimulation. Afferent axons from sensory neurons, whose cell bodies are located in the dorsal root ganglion (DRG), carry information from peripheral sensory receptors to the central neuron ${ }^{[6]}$. DRG is an aspiring part in the development of NP; DRG stimulation has multiple impacts on the abnormal changes that occur within the DRG as a result of peripheral afferent fiber injury ${ }^{[7]}$. The cell bodies of the DRG neurons are separated from each other, lying outside of the blood-nerve barrier ${ }^{[6]}$. DRG can play a crucial part in signal processing and transmission, which are essential for pain ${ }^{[8]}$.

There are some special ion channels and receptors, such as the transient receptor potential (TRP), P substance, glutamate receptor, and potassium, calcium, sodium channels ${ }^{[9]}$. During the progress of NP, the C-fibre neurons can cause pain ${ }^{[10,11]}$. The DRG can deliver the stimulus to the sensory neurons; and afferent axons from sensory neurons carry information from peripheral sensory receptors to the central neurons ${ }^{[6,12]}$. The TRP KO mice testified the function of TRP in the development of NP. Compared with the wildtype mice, the KO mice were more sensitive to the mechanical pain ${ }^{[13]}$. The TRP family is broadly reflected in the nervous system. TRP vanilloid 1 (TRPV1), TRP vanilloid 2 (TRPV2), TRP vanilloid 3 (TRPV3), TRP vanilloid 4 (TRPV4), TRP ankyrin 1 (TRPA1) and TRP melastatin 8 (TRPM8), which belong to the large TRP ion channel superfamily, are critical contributors to normal and pathological pain ${ }^{[14,15]}$. Smith et al. concluded that there was increased expression of TRPA1, TRPV1 and TRPM8 in DRG neurons ${ }^{[16]}$. Pan et al. reported that the TRPM8 and TRPA1 expression were up-regulated in both acute and chronic pain $^{[14]}$. Ceppa et al. considered that TRPV4 could contribute to pain and TRPA1 might contribute to inflammation ${ }^{[15]}$. TRPV3 is coded for by an open reading frame, transcribed from a gene adjacent to TRPV1, and is structurally homologous to TRPV ${ }^{[16]}$. TRPV1 and TRPV2 are first isolated from a cDNA library from rat sensory neurons ${ }^{[17,18]}$. Generally speaking, the TRP ion channels are widely linked to the NP.

Danggui Sini decoction (DGSD), a traditional Chinese medicine formula, has been widely used as a remedy for NP and other diseases in recent years ${ }^{[19]}$. DGSD consisted of 7 herbs ${ }^{[20]}$ : Angelica sinensis (12 g), Cassia twig (9 g), Paeonia lactiiflora (9 g), Tetrapanax papyriferus (6 g), Asarum (3 g), Glycyrrhiza uralensis (6 g) and Ziziphus jujuba Mill (10 g). Cheng et al. found that DGSD mediated the abnormal metabolic pathways, which could help treat rheumatoid $\operatorname{arthritis}^{[21,22]}$. The main ingredients of DGSD are ferulic acid, glycyrrhizin, cinnamic acid, cinnamaldehyde, z-ligustilide, hederagenin, paeoniflorin, albiflorin, sitosterol, ascorbate. DGSD is used to treat arthritis, diabetic peripheral neuropathy and coronary atherosclerotic heart disease. Gao et al. deemed that a mixture of glycyrrhizin, cinnamic acid and ferulic acid can protect the myocardium from the ischemiareperfusion injury ${ }^{[23]}$. Ferulic acid and z-ligustilide relieved the cold-induced vasospasm partly via TRPM8 and TPRA1 pathway ${ }^{[24,25]}$. Ferulic acid has ameliorative potential in mitigation of the pain associated with vincristine-induced painful neuropathy ${ }^{[26]}$. In this investigation based on an assumption that the composition and the thermodynamic properties in DGSD might restrain the cold-sensitive TRP channel, while cold-natured herbs might promote. Literature revealed that some constituents in DGSD could calm pain. This investigation comprehensively sought the role of temperature-sensitive TRP channel in NP; and explored the therapeutic effect of DGSD on NP as well as its impact on the TRP pathway.

\section{MATERIALS AND METHODS}

DGSD instant granules were obtained from Guangzhou Oversea Ology co. Ltd. Absolute ethyl alcohol and xylene were obtained from China Pharmaceutical Group Chemical Reagent Co. Ltd (Shanghai, China). Haematoxylin and eosin stain, PBS buffer, paraformaldehyde (4\%), propyl ketone, saccharose, Trionx, TBS, and Balsam neutral were acquired in Saiguo Biological Technology Co. Ltd (Shenzhen, China). Polyclonal rat antirabbit TRPV1-4, TRPA1, TRPM8 (ab6166, ab6183, ab85022, ab39260, ab58844, ab3243), and WB antibody diluent WB antibody were obtained from Abcam (Cambridge, UK), while PageR $\mu$ Ler prestained protein ladder and marker were from Thermo (Waltham, US). These were used for western-blot and immunohistochemistry. PVDF membrane was procured from Millipore (Billerica, US). And BCA protein content test kit, as well as RIPA, purchased from Biyun Tianye Biotechnology Co., Ltd (Shanghai, China).

\section{Experimental animals:}

Male Sprague Dawley rats (SD, 250-280 g, 7-8 w old) were purchased from the Experimental centre of Guangzhou University of Chinese medicine (License 
no. SYXK 202-0117; Guangzhou, China). Four rats were housed in individual cages under controlled room temperature $\left(20-22^{\circ}\right)$ and humidity (40-60\%), with alternative $12 \mathrm{~h}$ light/dark cycle in animal experimental centre of Medical College of Jinan University SPF animal housing management. Rats were acclimatized to the laboratory conditions for $1 \mathrm{w}$ before starting the experiments and were provided food and water ad libitum. All animal studies conformed to the Regulations of Experimental Animal Administration promulgated by the State Committee of Science and Technology of the People's Republic of China on November 14, 1988.

\section{The NP model $(\mathrm{CCI})^{[27]}$}

Rats ( $\mathrm{n}=128)$ were anaesthetized with sodium pentobarbital (3\%, $40 \mathrm{mg} / \mathrm{kg}$, ip). The sciatic nerve was exposed at the level of the middle of the thigh by blunt dissection through biceps femoris. Proximal to the sciatic was tied loosely (using 4.0 suture) around it 4 times with about $1 \mathrm{~mm}$ spacing under the dissection microscope, while 32 rats did not get nerve ligation, but were exposed to surgery. Then, gentamicin $(2 \mathrm{ml}$, $10 \mathrm{mg} / \mathrm{ml}$, im) was given after the suture of the wound.

\section{Behavior tests:}

Cold hyperalgesia was estimated by application of acetone and cold plate. About $100 \mu \mathrm{l}$ of acetone was released via a $100-\mu 1$ pipette onto the plantar surface of each hind paw ${ }^{[28]}$. Acetone was expelled out with forcing air through, thereby avoiding mechanical stimulation of the paw with the pipette ${ }^{[29]}$. Total time lifting or clutching each hind paw was recorded with a maximum cutoff time of $120 \mathrm{~s}$. Approximately $30 \mathrm{~min}$ after cold acetone, the lateral plantar surface was exposed to a cold plate; the withdrawal latency and duration were recorded. Cold stimulation was repeated 3 times at an interval of 10 min for each paw and the mean was calculated. Mechanical allodynia was assessed with von Frey filaments ${ }^{[30,31]}$. The plantar surface of each hind paw was stimulated 10 times with each filament (2-30 g), starting with the $2 \mathrm{~g}$ filament and increasing until the rats responded by clutching and/or lifting the paw off the surface of the test apparatus. Paw lifting was coded as a positive response. Once a positive response was detected, sequentially lower weight filaments were used to estimate the sensory threshold for each paw. Then, the pain threshold was calculated. Heat hyperalgesia was evaluated by hot plate experiment. The lateral plantar surface was exposed to the heated plate. The first withdrawal latency and duration were recorded. The heat stimulation was replicated 3 times at an interval of $10 \mathrm{~min}$ for each paw and the mean was calculated.

\section{Groups:}

One hundred and twenty eight SD rats were separated randomly into 4 groups, the sham-operated group (SG), CCI model group (CG), pregabalin group (PG), and DGSD-treated group (DG). The rats in SG underwent surgery, but no nerve was ligated. All drugs were administered orally for $21 \mathrm{~d}$. Given the surface area ratio of human to rats, $0.1 \mathrm{~g}$ DGSD was dissolved in normal saline, which could be irrigated to the rats of DG in $8 \mathrm{ml} / \mathrm{kg} / \mathrm{d}$, bid. Rats in SG and CG were gavaged with saline $8 \mathrm{ml} / \mathrm{kg} / \mathrm{d}$, bid. Rats in PG were given pregabalin $(10 \mathrm{mg} / \mathrm{kg} / \mathrm{d}$, bid). Besides, each group was subdivided into $4,7,14,21 \mathrm{~d}$ of the group of time, there were 8 rats in each time group. Cold acetone and detection plate cold hyperalgesia, Von Frey test for the detection of mechanical allodynia and thermal hyperalgesia detection of hot plate experiments were conducted.

\section{Hematoxylin-eosin (HE) stain:}

Ligation of the sciatic nerve was stored in $4 \%$ paraformaldehyde for $24 \mathrm{~h}$ and then embedded in paraffin and sectioned in accordance to routine procedures ${ }^{[32]}$. After being deparaffinized with xylene, sections were dehydrated with ethanol (100, 90, 80 and $70 \%$, respectively) and then rinsed in PBS for $5 \mathrm{~min}$. Then a pathologist who is blinded to experiments assessed the severity of tissue damage by HE staining to evaluate the inflammatory injury and neurochemical change.

\section{Immunohistochemistry in rat DRG tissues:}

DRGs were fixed in $4 \%$ paraformaldehyde with $0.1 \%$ picric acid for $24 \mathrm{~h}$ and then transferred into a $20 \%(\mathrm{w} / \mathrm{v})$ sucrose solution for cryoprotection ${ }^{[33]}$. DRGs were sectioned at $9 \mu \mathrm{m}$ utilizing a standard cryostat. Sections from individual animals were homogenized in sodium citrate antigen repair solution (1:1000 attenuation, $\mathrm{pH}=7)$. Primary antibodies (1:200) were diluted in $5 \%$ NGS and $1 \%$ BSA with $0.2 \%$ Triton and incubated overnight at $4^{\circ}$. Primary antibodies used in this study are as follows, TRPV1, TRPV2, TRPV3, TRPV4, TRPA1 and TRPM8. After washing with PBS, the sections were incubated with the corresponding second antibody. Immune-stained 
sections were reviewed under a Leica fluorescence microscope, and images were captured with a CCD spot camera.

\section{Western blots:}

Western blot was used to determine the expression of TRPA1,TRPM8, TRPV1-4 in offside DRGs. DRGs (L4-L6), corresponding to the afferent pathway from the hind paw, were carefully removed and processed as previously described ${ }^{[34]}$. The DRGs were homogenized in RIPA cell lysis buffer, with the homogenates $(1.5 \mu \mathrm{l})$ were centrifuged at $14000 \mathrm{rpm}$ at $4^{\circ}$ for $10 \mathrm{~min}$. The supernatant was separated into 3 equal portions. The extracted protein solution and upper sample buffer were mixed in $4: 1$, and placed in $100^{\circ}$ boiling water bath to boil for $10 \mathrm{~min}$, cooled to room temperature, centrifuged at $3000 \mathrm{rpm}$ for $5 \mathrm{~min}$ and then stored in a $-20^{\circ}$ refrigerator. The samples were then loaded on $10 \%$ sodium dodecyl sulphate-polyacrylamide gels and transferred to polyvinylidene fluoride membranes. The blots were incubated with a primary antibody against TRPV1-4, TRPA1 and TRPM8, respectively overnight at $4^{\circ}$. After that, the sections were incubated with the corresponding second antibody at room temperature for $1 \mathrm{~h}$. In the meantime, the protein concentration was measured (BCA protein content test kit).

\section{Statistical methods:}

Image Analysis Software (Adobe, California, US), Grayscale analysis software (Alpha Innotech, Silicon Valley, US), fluorescence microscope (Leica, German) were employed to acquire, film, observe the image. All data were statistically processed using SPSS13.0. The experimental data of normal distribution are expressed as mean \pm standard deviation, compared between the 2 groups by independent T-test. The mean values of the bleaching protocols in each session were analysed by one-way ANOVA supplemented by SNK or Tamhane's test. All analyses were performed at the $5 \%$ significance level.

\section{RESULTS AND DISCUSSION}

Cold plate experiments (fig. 1A and B) and the results of the acetone experiment (fig. 1C) indicated that cold pain sensitivity and cold pain in CG appeared in $\mathrm{d} 4$, while they were enhancing during 7-10 d, reaching the highest peak d 14, yet, declining by d 21. Compared to $\mathrm{SG}$, these variations have statistical criteria $(4,7$, $14,21 \mathrm{~d}, \mathrm{p}<0.05)$. DGSD decreased the hyperalgesia and allodynia obviously between $\mathrm{d}$ 4-21 $(\mathrm{p}<0.05)$ especially on $\mathrm{d} 7$ and 14. The effect of DGSD on the pain was superior to the curative effect of PG $(14,21 \mathrm{~d}$, $\mathrm{p}<0.05$; Table 1).

The sequels manifested that the rats in CG appeared to be in apparent mechanical pain sensitivity on the postoperative $\mathrm{d} 4$, however the mechanical pain threshold on $\mathrm{d} 4$ was significantly reduced. On d 14, the pain threshold (PWL) reached a minimum, and then increased (4-21 $d \mathrm{p}<0.05)$. DGSD could relieve the mechanical pain sensitivity of NP rats more obviously than pregabalin (4-21 d). Furthermore, DGSD analgesic effect continues to increase (fig. 1D, Table 2).

Compared to the SG, CG rats showed an obvious decrease of thermal pain threshold on the postoperative $\mathrm{d} 4$, a decrease by the $\mathrm{d} 14$ to the lowest level, and a pain threshold at $d 21$. DGSD compared to pregabalin, had a more pronounced effect on the CCI model of thermal hyperalgesia $(14,21 \mathrm{~d}, \mathrm{p}<0.05$; fig. $1 \mathrm{E}$, Table 3).

On the d 14 after CCI operation, the inflammatory cell infiltration was observed in the $\mathrm{CG}$, moreover the structure of the sciatic nerve was destroyed, some neuronal cells were devastated, while the sections in PG were no different from those in the CG. And the inflammatory factors were substantially reduced in DGSD, and a small number of regenerated Schwann cells also appeared (fig. 2).

From the immunofluorescence study results, the expression of temperature-sensitive TRP channel protein in the DRG of $\mathrm{CG}$ rats altered in varying degrees at different time points. Cold-stimulated receptor channel proteins TRPM8 and TRPA1, which the expression of TRPM8 and TRPA1 increased over time, reached the highest peak on $\mathrm{d} 14$. The explicit expression of TRPM8 commenced lower on $\mathrm{d} 21$ after the surgical operation. The expression of TRPV1 and TRPV4 continued to increase on $\mathrm{d} 7$. The decisive expression of TRPV1 and TRPV4 increased to the highest level on $\mathrm{d} 14$, and decreased on $\mathrm{d} 21$. The expression of TRPV1 and TRPV4 protein, which is sensitive to high temperatures increased sharply from d 4 after operation. The increasing was initiated on the $\mathrm{d} 7$, while the expression increased to the highest level on d 14, and decreased on d 21. In immunofluorescence studies also, the distribution of positive sites of TRPV1 and TRPV4 in the CG was also apperceived, and the TRPV1 distributed in small diameter neurons rather the large diameter neurons, whereas TRPV4 spread 
A
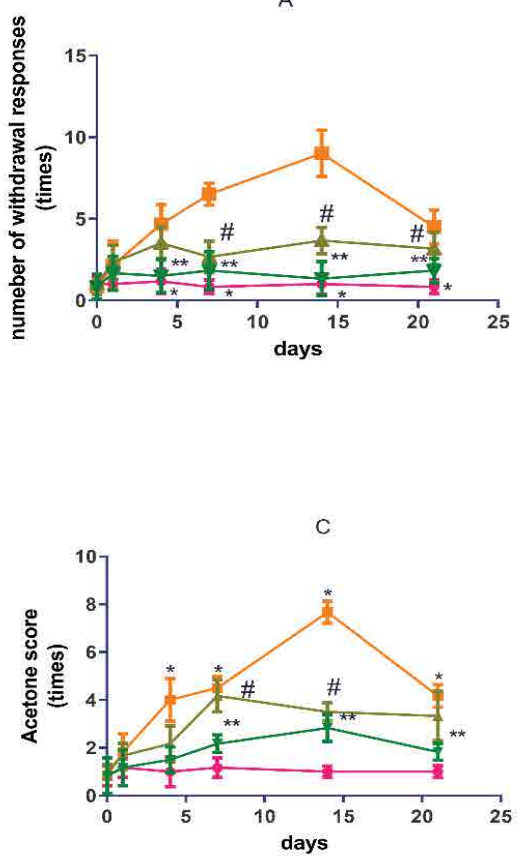

E

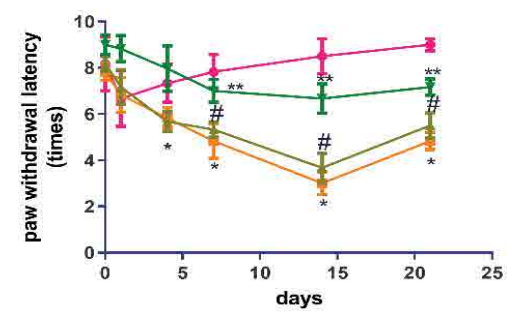

$B$

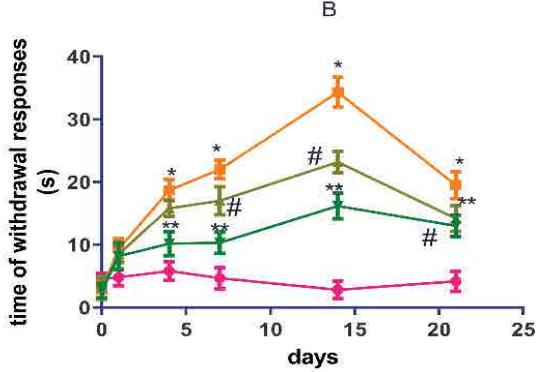

D

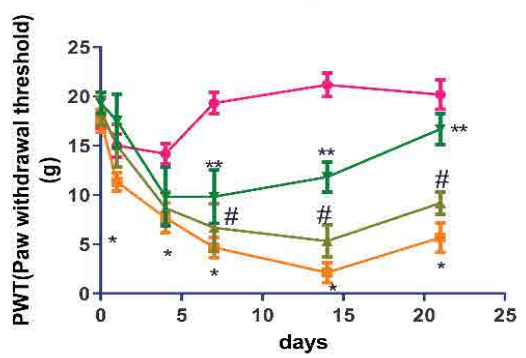

Fig. 1: Cold hyperalgesia estimated by cold detection plate and cold acetone experiment

A. shows the frequency of paw withdrawal from day one to day 21 . B. indicates the total time lifting or clutching the hind paw from the experiment to the end of the experiment. C. states the results of the acetone experiment $(p<0.05)$. D: manifests frequency of paw withdrawal. E: demonstrates the PWL. *States the contrast of SG and CG, **means the comparison of DG and CG, while \#demonstrates the comparison between DG and PG. (-०-) SG, (- - C CG, (- $-1-)$ PG, $(-\nabla-)$ DG

TABLE 1: COMPARISON OF COLD ALLODYNIA BEHAVIOR IN DIFFERENT GROUPS OF RATS AT DIFFERENT TIME POINTS

\begin{tabular}{|c|c|c|c|c|c|c|}
\hline & Group & $1 \mathrm{~d}$ & $4 \mathrm{~d}$ & $7 \mathrm{~d}$ & $14 \mathrm{~d}$ & $21 d$ \\
\hline \multirow{4}{*}{ Number of withdrawal responses (times) } & SG & $1.00 \pm 0.02$ & $1.17 \pm 0.75$ & $0.83 \pm 0.41$ & $1.00 \pm 0.63$ & $0.83 \pm 0.41$ \\
\hline & CG & $2.17 \pm 1.47$ & $4.67 \pm 1.21^{*}$ & $6.50 \pm 0.66^{*}$ & $9.00 \pm 1.41$ & $4.50 \pm 1.05^{*}$ \\
\hline & PG & $2.33 \pm 1.03$ & $3.50 \pm 1.05$ & $2.67 \pm 0.97$ & $3.67 \pm 0.82^{\#}$ & $3.17 \pm 0.98^{* *}$ \\
\hline & DG & $1.67 \pm 1.03$ & $1.50 \pm 1.05^{* *}$ & $1.83 \pm 1.17^{* *}$ & $1.34 \pm 1.03^{* *}$ & $1.83 \pm 0.75^{* *}$ \\
\hline \multirow{4}{*}{ Time of withdrawal responses (s) } & SG & $4.83 \pm 1.35$ & $5.83 \pm 1.52$ & $4.67 \pm 1.70$ & $2.83 \pm 1.40$ & $4.17 \pm 1.62$ \\
\hline & CG & $9.33 \pm 1.73$ & $18.67 \pm 1.75^{*}$ & $22.00 \pm 1.52^{*}$ & $34.3 \pm 2.42^{*}$ & $19.50 \pm 2.14^{*}$ \\
\hline & PG & $8.50 \pm 1.43$ & $15.83 \pm 1.32$ & $17.00 \pm 2.25^{\#}$ & $23.2 \pm 1.71^{* *}$ & $14.17 \pm 2.04$ \\
\hline & DG & $8.17 \pm 2.14$ & $10.17 \pm 1.93^{* *}$ & $10.33 \pm 1.73^{*}$ & $16.2 \pm 2.04^{* *}$ & $13.00 \pm 1.74^{* *}$ \\
\hline \multirow{4}{*}{ Acetone score (times) } & SG & $1.17 \pm 0.41$ & $1.00 \pm 0.63$ & $1.17 \pm 0.41$ & $1.00 \pm 0.24$ & $1.00 \pm 0.26$ \\
\hline & CG & $1.83 \pm 0.75$ & $4.00 \pm 0.89^{*}$ & $4.50 \pm 0.49^{*}$ & $7.67 \pm 0.46^{*}$ & $4.17 \pm 0.47^{*}$ \\
\hline & PG & $1.67 \pm 0.52$ & $2.17 \pm 0.75$ & $4.17 \pm 0.67^{\#}$ & $3.50 \pm 0.38^{* *}$ & $3.33 \pm 1.03^{\#}$ \\
\hline & DG & $1.17 \pm 0.75$ & $1.50 \pm 0.55^{* *}$ & $2.17 \pm 0.37^{* *}$ & $2.83 \pm 0.57^{* *}$ & $1.83 \pm 0.35^{* *}$ \\
\hline
\end{tabular}

*States the contrast of SG and CG, ${ }^{* *}$ means the comparison of DG and CG, while "demonstrates the comparison between DG and PG

over the large diameter neurons. Similarly, TRPV2 and TRPV3 expression in DRG overall trend were further increased and TRPV2 and TRPV3 on d 4 also showed a significant increase trend. TRPV2 positive expression reached its peak on $\mathrm{d} 7$, declined on $\mathrm{d} 14$, and the expression of TRPV 3 on $\mathrm{d} 14$ relative to that on d 7 decreased, but the definite expression on $\mathrm{d} 21$ rose to a small peak (fig. 3). 
The expression of TRPM8 and TRPA1 in DRG increased evidently on $\mathrm{d} 4$ after the operation in the CG. The protein levels of TRPM8 and TRPA1 continued to increase on $\mathrm{d} 7$, reaching the highest level on d 14, while it decreased on d 21. DGSD restrained the expression of TRPM8 and TRPA1 at 4, 7, 14 and $21 \mathrm{~d}(\mathrm{p}<0.05)$, and the suppressive effect was more pronounced as the time passed. That was to say, DGSD could down-regulate TPRM8 and TRPA1 expression. The pregabalin analogously had an inhibitory effect on the abnormally increased TRPM8 protein, but the pregabalin did not curb TRPA1 distinctly ( $p>0.05$ ). TRPV1, TRPV2, TRPV3 and TRPV4 were remarkably having high levels of expression in each pathological period of $\mathrm{CG}$. In $\mathrm{CG}$, the content of TRPV1, TRPV2, TRPV3 and TRPV4 protein in DRG increased markedly on $\mathrm{d} 4$ and since then all are in a highly perverted state of expression. However, DGSD repressed TRPV1-TRPV4 protein expression on $\mathrm{d} 4,7$, 14 and $21(\mathrm{p}<0.05)$. Pregabalin had a certain inhibitory effect on TRPV1 channel $(4,7,14,21 \mathrm{~d}, \mathrm{p}<0.05)$, but no obvious inhibition on the TRPV2-V4 pathway (fig. 4).

Chronic constriction injury of the sciatic nerve is the animal model of peripheral NP selected in this experiment, and the advantages being less damage to the rats, superior model for stability, and can simulate the clinical pathological process and symptoms of NP patients. CCI model was established in 1988 by Bennett et al. The rats developed blatant cold, heat and mechanical hyperalgesia and spontaneous pain on the d3-4 afterCCI surgery, then the pain was aggravated from d 7 to 10 , reaching the peak on $\mathrm{d} 14$ and subsequently, the sensitivity of the pain was reduced. The operation that the sciatic nerve of rats was ligated 4 times loosely was constructed under the microscope, while the ligation did not block the adventitia of the sciatic nerve. Rats in the SG demonstrated increased mechanical hyperalgesia on d 1-3 after surgery, considered as a surgical wound caused, for the pain disappeared after $\mathrm{d} 3$. The right lower limb of the CG exhibited apparent cold, heat and mechanical hyperalgesia on $d 4$, the hyperalgesia was more pronounced on $\mathrm{d} 7$ to 10 and by d 14 it was at its peak, after which the pain started to decrease. All are essentially consistent with what was described in the literature ${ }^{[29]}$. Experimental animals did not develop ulceration of the limbs, autophagy or other abnormalities, indicating that the model development was successful.

Studies have manifested that DGSD could relieve diabetes-induced $\mathrm{NP}^{[35]}$. On the basis of cold plate test and acetone test, DGSD could visibly relieve cold pain sensitivity in CG rats on $\mathrm{d} 7,14$ and 21, and pregabalin could alleviate the pain to certain extent, but it was not comparable to that produced by DGSD. DGSD, as well as pregabalin, could alleviate the mechanical hyperalgesia. As for heat hypersensitivity, DGSD demonstrated greater ability to reduce heat pain compared to pregabalin. Therefore, DGSD appeared to not only relieve cold pain but also mechanical pain and heat pain. Given the HE-stained sections, the ligated sciatic nerve patch showed very high inflammatory cell infiltration, greater damage to the structure of nerve tissue and even the destruction of nerve cell morphology.

TRPM8 and TRPA1 channel can be activated by cold temperatures or by a cooling agent ${ }^{[36,37]}$, which result

TABLE 2: COMPARISON OF MECHANICAL ALLODYNIA BEHAVIOR OF RATS IN DIFFERENT GROUPS AT DIFFERENT TIME POINTS

\begin{tabular}{|c|c|c|c|c|c|c|}
\hline & Group & $1 \mathrm{~d}$ & $4 d$ & $7 d$ & $14 d$ & $21 d$ \\
\hline $\begin{array}{l}\text { MWT } \\
\text { (g) }\end{array}$ & $\begin{array}{l}\text { SG } \\
\text { CG } \\
\text { PG } \\
\text { DG }\end{array}$ & $\begin{array}{l}15.00 \pm 1.16 \\
11.33 \pm 0.94 \\
15.00 \pm 2.16 \\
17.50 \pm 2.74\end{array}$ & $\begin{array}{c}14.17 \pm 1.04 \\
7.67 \pm 1.51^{*} \\
8.67 \pm 1.63^{* *} \\
9.83 \pm 2.99\end{array}$ & $\begin{array}{c}19.33 \pm 1.08 \\
4.67 \pm 1.03 \\
6.67 \pm 2.42^{\#} \\
9.83 \pm 2.71^{* *}\end{array}$ & $\begin{array}{c}21.17 \pm 1.22 \\
2.13 \pm 1.03^{*} \\
5.33 \pm 1.63^{* *} \\
11.83 \pm 1.54^{* \#}\end{array}$ & $\begin{array}{c}20.17 \pm 1.49 \\
5.67 \pm 1.51^{*} \\
9.17 \pm 1.13^{* *} \\
16.67 \pm 1.58 \\
* * \#\end{array}$ \\
\hline
\end{tabular}

*States the contrast of SG and CG, **means the comparison of DG and CG, while "demonstrates the comparison between DG and PG

TABLE 3: COMPARISON OF THERMOPATHIC BEHAVIORAL BEHAVIOR OF RATS IN DIFFERENT GROUPS AT DIFFERENT TIME POINTS

\begin{tabular}{lcccccc}
\hline & Group & 1 d & 4d & 7 d & 14 d & 21 d \\
\hline & SG & $6.67 \pm 1.21$ & $7.33 \pm 0.82$ & $7.83 \pm 0.75$ & $8.50 \pm 0.76$ & $9.00 \pm 0.27$ \\
PWL & CG & $6.83 \pm 0.75$ & $5.83 \pm 0.45^{*}$ & $4.83 \pm 0.75^{*}$ & $3.00 \pm 0.49^{*}$ & $5.83 \pm 0.45^{*}$ \\
$(S)$ & PG & $7.17 \pm 0.75$ & $5.67 \pm 0.43^{* *}$ & $5.33 \pm 0.33^{* *}$ & $3.67 \pm 0.63^{* *}$ & $5.50 \pm 0.55^{* *}$ \\
& DG & $8.83 \pm 0.57$ & $7.97 \pm 0.98^{* *}$ & $7.00 \pm 0.49^{* *}$ & $6.67 \pm 0.63^{* * \#}$ & $7.17 \pm 0.35^{* * \#}$ \\
\hline
\end{tabular}

*States the contrast of SG and CG, ${ }^{* *}$ means the comparison of DG and CG, while "demonstrates the comparison between DG and PG 

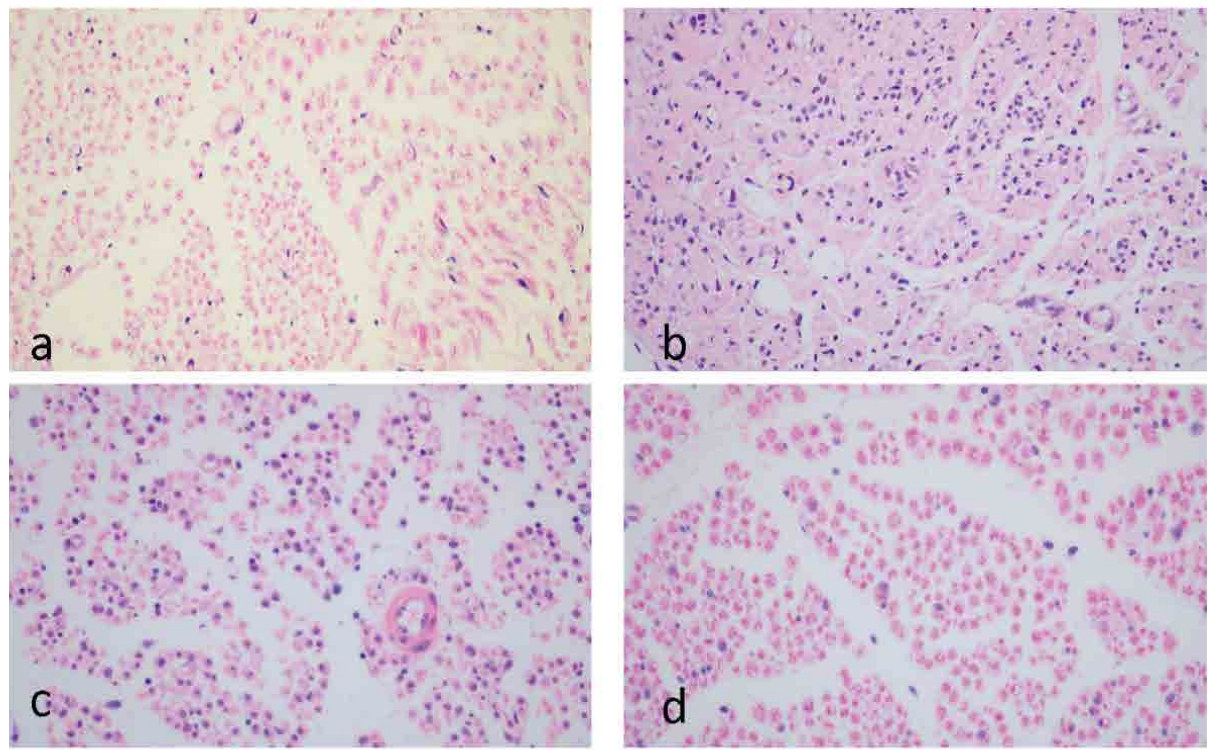

Fig. 2: Hematoxylin-eosin (HE) staining of rat sciatic nerve

(a) Rats in the sham-operated group had intact nerve structure and no inflammatory cell infiltration. (b) The destruction of the nerve structure and the neutrophil infiltration were observed in pathological sections taken from CCI model mice. (c) Pregabalin relieved pain but failed to reconstruct neural structure, and neutrophil infiltration was observed in sections from rats of PG. (d) DGSD obviously decreased infiltration of inflammatory cells. All figures were taken at $400 \times$ microscope

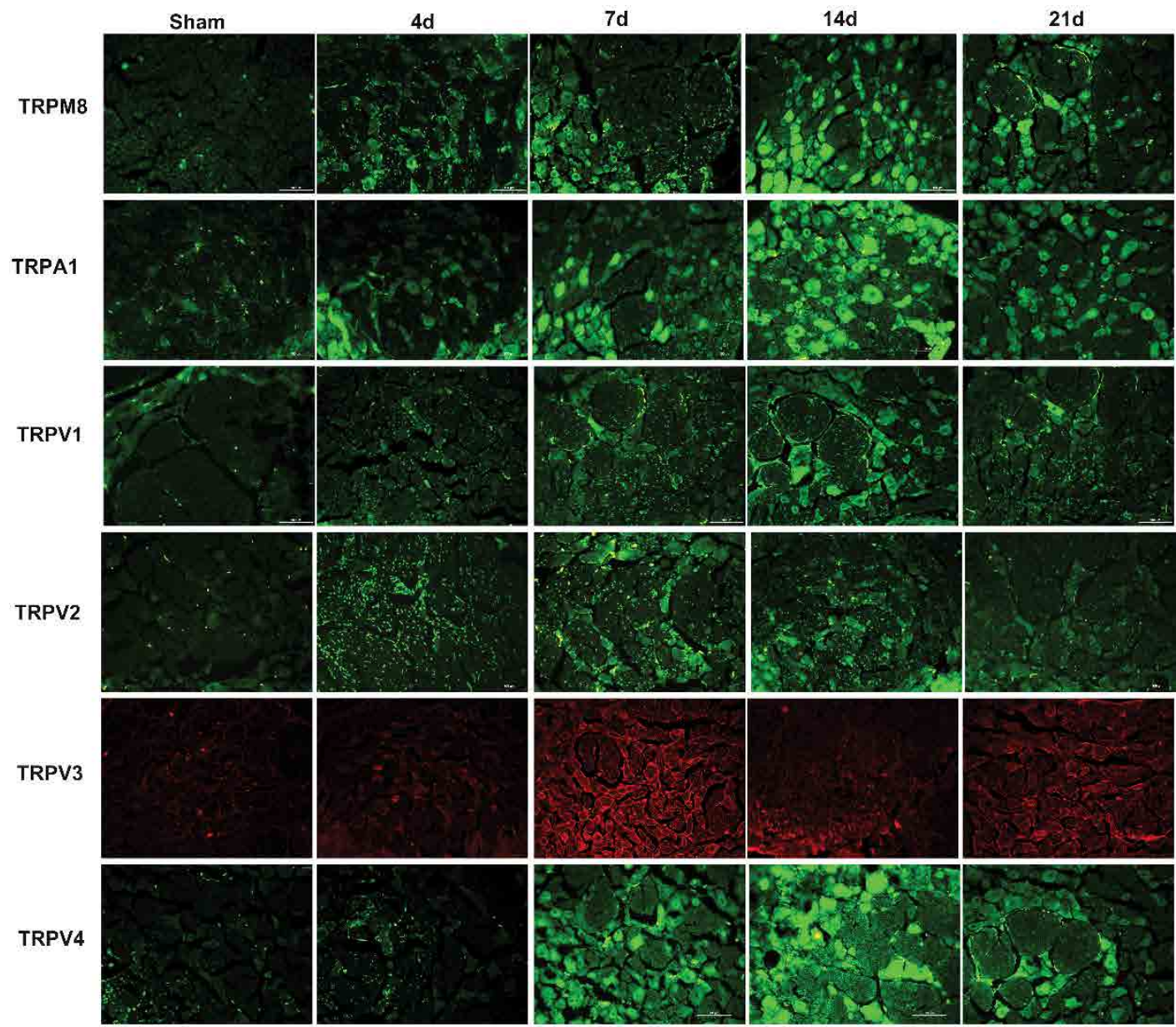

Fig. 3: Immunofluorescence stain of DRG temperature-related TRP channel

Sham- sham-operated group (SG), 4 d CCI- d 4 CG, 7 d- d 7 of CG, 14 d- d 14 of CG, 21 d- the d 21 of CG. The graphs above showed the expression of TRPM8, TRPA1, and TRPV1-V4 in the sham operation group, CCI model group. Green and red fluorescence were on behalf of positive cells. All figures were taken at $200 \times$ microscope 

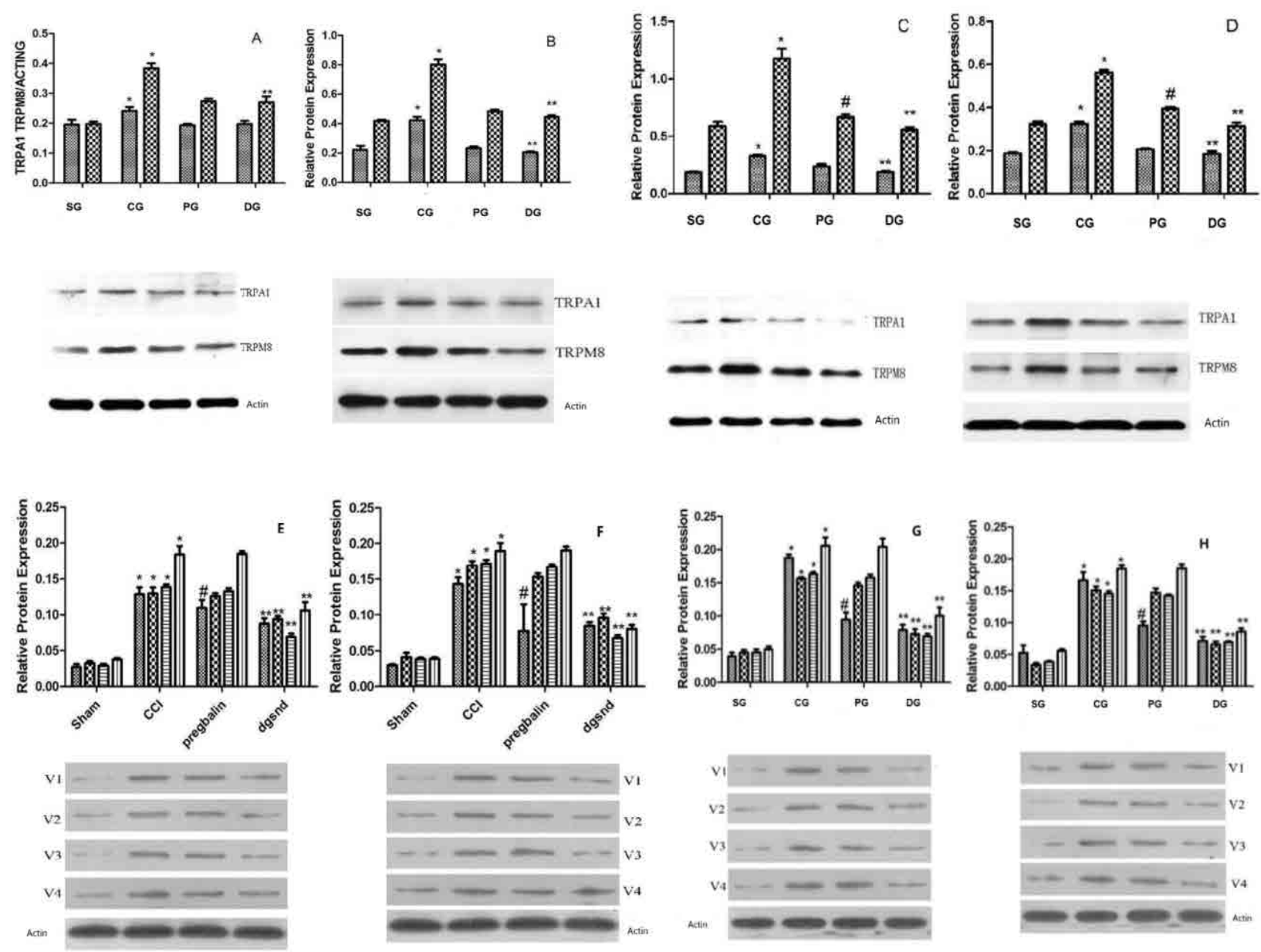

Fig. 4: TRP pathway protein expression

*States the contrast of SG and CG, **means the comparison of DG and CG, while *demonstrates the comparison between DG and PG. It could be observed that the content of proteins of TRPM8 and TRPA1 on the (A) d 4, (B) d 7, (C) d 14 and (D) d 21, ( $\because$ TRPA1 and $\infty$ TRPM8). Compared with the CG, DGSD refrained the expression of TRPM8 and TRPA1 at 4, 7, 14 and $21 \mathrm{~d}$ (p<0.05). It could be observed that the content of proteins of TRPV1, TRPV2, TRPV3, and TRPV4 on the (E) d 4, (F) d 7, (G) d 14,

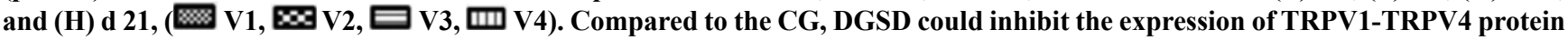
at $4,7,14$ and $21 \mathrm{~d}(\mathbf{p}<0.05)$

in $\mathrm{NP}^{[38,39]}$. The temporal and spatial abnormalities of TRPM8 and TRPA1 in DRG were detected by immunofluorescence, and up-regulation of DRG TRPM8 and TRPA1 channel proteins was observed on site of the NP model. This can also be confirmed by the Western blot. Cold-stimulated receptor channel proteins TRPM8 and TRPA1 were consistent with the tendency of neuropathic cold pain sensitivities, most likely involved in the pathogenesis of NP and were an important factor mediating cold hyperalgesia. Also, increased levels of DRG TRPM8 RNA and TRPA1 RNA were detected at the genetic level in injured ipsilateral NP ${ }^{[40]}$. When TRPM8 and TRPV1 agonists were intrathecally injected, cold pain sensitization was increased in NP rats; while TRPM8 and TRPA 1 blockers alleviated the cold pain sensitization ${ }^{[41,42]}$. TRPA1 both as a cold receptor stimulus, but also as a sensitizer of mechanoreceptors, was involved in the pathogenesis of NP mechanical hyperalgesia; TRPA1 knockout mice were less sensitive to mechanical stimuli as the normal ${ }^{[42]}$. TRPA1 antagonist subcutaneous injection can obviously relieve mechanical pain sensation, while intrathecal injection of the antagonist markedly suppressed the allodynia ${ }^{[43]}$. All these demonstrated that TRPM8 and TRPA1 were involved in the pathological process of NP, especially cold hyperalgesia. DGSD could down-regulate the expression of TRPM8 and TRPA1, specifically the TRPM8, than pregabalin, which might be the underlying mechanism that DGSD could treat NP.

As members of the TRP, TRPV1-4 are activated by distinct heat temperatures ${ }^{[44]}$. TRPV1 and TPRV4 are activated by mechanical stimuli as well, and are, therefore, closely associated with 
mechanical hyperalgesia and thermal hyperalgesia. Amaya et al. considered that inflammatory pain could cause an increase in TRPV1 expression ${ }^{[44]}$. Hong et al. demonstrated that TRPV1 expression was increased in large myelinated A-fibre DRG neurons, while it was decreased in small unmyelinated C-fibre neurons as the NP develops ${ }^{[45]}$. Barabas et al. deemed that abnormal expression of TRPV1 in the periphery of the skin could promote the release of the SP in DRG, thereby causing neurogenic inflammation ${ }^{[46]}$. In the central end of the spinal dorsal horn, TRPV1 is highly expressed as an important calcium source, thus promoting the release of pain neurotransmitters ${ }^{[4]}$. A single intrathecal injection of TRPV1 antagonists relieved thermal pain and mechanical hyperalgesia induced by peripheral nerve injury; the analgesic effect was more pronounced with successive multiple intrathecal and high doses of its antagonist ${ }^{[48,49]}$. Moreover, intrathecal injection of TRPV1-specific antagonists can relieve pain caused by sciatic nerve ligation ${ }^{[50,51]}$. Physiologically, TRPV1 is mainly expressed in small and medium-sized neuronal cells, and TRPV1 expression increased in diabetic neuralgia model and mainly distributed in small DRG neurons ${ }^{[52]}$. For hyperalgesia-induced model of paclitaxel chemotherapy, Hara et al. found in DRG TRPV1 held the characteristics of a volume-dependent redistribution ${ }^{[53]}$. In the $S G$, it was found that TRPV1 was mainly expressed in small diameter neurons; that is, TRPV1 was distributed in small diameter neurons under physiological conditions, which was consistent with previous studies. Initially, TRPV1 expression was increased in small diameter neuronal cells in the CG; as time went by, at the $\mathrm{d} 14$ and $\mathrm{d} 21$, the TRPV1 expression tended to gradually distribute from small diameter neurons to large and medium diameter neurons, which was not the same as the previous study. Preceding generations might not observe the expression of TRPV1 over time, which could be responsible for this. Results of imunofluorescence indicated that the expression of TRPV1 protein channel was similar to the tendency of thermal hyperalgesia. Therefore, it is believed that TRPV1 may be a determine factor in thermal hyperalgesia ${ }^{[54]}$. Frederick et al. found increased expression of TRPV2 mRNA on the ipsilateral DRG of the injured nerve ${ }^{[38]}$. Increased expressions of TRPV2 and TRPV3 in rat DRG operated side, which was not consistent with the specific trends of mechanical and thermal hyperalgesia, but overall they were comparable, were similar to previous research. Zhang et al. detected that TRPV4 mRNA content in
DRG was considerably increased in all DRG chronic pain models (CCD) from $\mathrm{d} 7$ to $\mathrm{d} 28$ postoperatively ${ }^{[54]}$. Intrathecal injection of colchicine, TRPV4 antagonists, could down-regulate the expression of TRPV4 protein mRNA and alleviated the thermal hyperalgesia in CCD rats ${ }^{[55]}$. In this experiment, increased expression TRPV4 was consistent with the tendency of TRPV1, which verified the previously reported results ${ }^{[55]}$. And the distribution of TRPV4 tended toward larger diameter neurons in immunofluorescence. From the above, TRPV1, TRPV2, TRPV3 and TRPV4 were all involved in the development of NP in the CCI model, which might be a decisive element inducing thermal hyperalgesia and mechanical hyperalgesia. Pinnacle expression TRPV2 and TRPV 3 is on $\mathrm{d} 7$, indicating that TRPV2 and TRPV3 probably play a major role in the early stage of pain, while the level of TRPV1 and TRPV4 reached the highest peak on $\mathrm{d} 14$, indicating that TRPV1 and TRPV4 mediate pain later in CG NP. Therefore, we can hold that the restorative effect of DGSD on thermal hyperalgesia and mechanical hyperalgesia in CG rats may be realized by downregulating the expression of TRPV1-TRPV4.

DGSD cannot only suppress the cold allodynia of NP of the $\mathrm{CG}$ rats, but also alleviate its mechanical allodynia and thermal hyperalgesia. TRPV1, TRPV2, TRPV3, TRPV4, TRPM8, and TRPA1 are involved in the pathological process of NP, while TRPM8 and TRPA1 might be responsible for cold hyperalgesia. As for the pathological processes of mechanical hyperalgesia and thermal hyperalgesia, TRPV2 and TRPV3 may probably play a major role in the early stage, and TRPV1 and TRPV4 could mediate pain later. DGSD can down-regulate abnormally high expression of temperature-dependent TRP channel protein.

\section{Conflict of interest:}

The author(s) confirm that this article content has no conflict of interest.

\section{Acknowledgements:}

This work was supported by the National Natural Science Foundation of China (Grant No. 81173189).

\section{REFERENCES}

1. Zhang ZJ, Jiang BC, Gao YJ. Chemokines in neuron-glial cell interaction and pathogenesis of neuropathic pain. Cell Mol Life Sci 2017;74(18):3275-91.

2. Nakagawa T. Sensory mechanism of pain and novel analgesics. Seikagaku 2013;85(7):561-5. 
3. Arendt-Nielsen L, Morlion B, Perrot S, Dahan A, Dickenson A, Kress HG, et al. Assessment and manifestation of central sensitisation across different chronic pain conditions. Eur J Pain 2018;22(2):216-41.

4. Feldman EL, Nave KA, Jensen TS, Bennett DLH. New Horizons in Diabetic Neuropathy: Mechanisms, Bioenergetics, and Pain. Neuron 2017;93(6):1296-313.

5. Krames ES. The Dorsal Root Ganglion in Chronic Pain and as a Target for Neuromodulation: A Review. Neuromodulation 2015;18(1):24-32.

6. Staaf S, Oerther S, Lucas G, Mattsson JP, Ernfors P. Differential regulation of TRP channels in a rat model of neuropathic pain. Pain 2009;144(1-2):187-99.

7. Suzuki N, Hasegawa-Moriyama M, Takahashi Y, Kamikubo Y, Sakurai T, Inada E. Lidocaine Attenuates the Development of Diabetic-Induced Tactile Allodynia by Inhibiting Microglial Activation. Anesth Analg 2011;113(4):941-6.

8. Liao YH, Zhang GH, Jia D, Wang P, Qian NS, He F, et al. Spinal astrocytic activation contributes to mechanical allodynia in a mouse model of type 2 diabetes. Brain Res 2011;1368:324-35.

9. Basbaum AI, Bautista DM, Scherrer G, Julius D. Cellular and Molecular Mechanisms of Pain. Cell 2009;139(2):267-84.

10. Bölcskei K, Helyes Z, Szabó A, Sándor K, Elekes K, Németh $\mathrm{J}$, et al. Investigation of the role of TRPV1 receptors in acute and chronic nociceptive processes using gene-deficient mice. Pain 2005;117(3):368-76.

11. Tominaga M, Julius D. Capsaicin receptor in the pain pathway. Jpn J Pharmacol 2000;83(1):20-4.

12. Moore C, Gupta R, Jordt SE, Chen Y, Liedtke WB. Regulation of Pain and Itch by TRP Channels. Neurosci Bull 2018;34(1):120-142.

13. Chukyo A, Chiba T, Kambe T, Yamamoto K, Kawakami $\mathrm{K}$, Taguchi K. Oxaliplatin-induced changes in expression of transient receptor potential channels in the dorsal root ganglion as a neuropathic mechanism for cold hypersensitivity. Neuropeptides 2018;67:95-101.

14. Pan Y, Chen F, Huang S, Cai Z, Lan H, Tong Y, et al. TRPA1 and TRPM8 Receptors May Promote Local Vasodilation that Aggravates Oxaliplatin-Induced Peripheral Neuropathy Amenable to 17 beta-Estradiol Treatment. Curr Neurovasc Res 2016;13(4):309-17.

15. Ceppa E, Cattaruzza F, Lyo V, Amadesi S, Pelayo JC, Poole DP, et al. Transient receptor potential ion channels V4 and A1 contribute to pancreatitis pain in mice. Am J Physiol Gastrointest Liver Physiol 2010;299(3):G556-71.

16. Smith GD, Gunthorpe MJ, Kelsell RE, Hayes PD, Reilly P, Facer P, et al. TRPV3 is a temperature-sensitive vanilloid receptor-like protein. Nature 2002;418(6894):186-90.

17. Gunthorpe MJ, Benham CD, Randall A, Davis JB. The diversity in the vanilloid (TRPV) receptor family of ion channels. Trends Pharmacol Sci 2002;23(4):183-91.

18. Caterina MJ, Schumacher MA, Tominaga M, Rosen TA, Levine JD, Julius D, et al. The capsaicin receptor: a heat-activated ion channel in the pain pathway. Nature 1997;389(6653):816-24.

19. El-Seedi HR, Burman R, Mansour A, Turki Z, Boulos L, Gullbo J, et al. The traditional medical uses and cytotoxic activities of sixty-one Egyptian plants: discovery of an active cardiac glycoside from Urginea maritima. J Ethnopharmacol 2013;145(3):746-57.

20. Wu JX, Zheng H, Yao X, Liu XW, Zhu HJ, Yin CL, et al. Comparative analysis of the compatibility effects of DangguiSini Decoction on a blood stasis syndrome rat model using untargeted metabolomics. J Chromatogr B Analyt Technol Biomed Life Sci 2019;1105:164-75.

21. Cheng B, Zheng $\mathrm{H}, \mathrm{Wu} \mathrm{F}, \mathrm{Wu}$ J, Liu X, Tang C, et al. Metabolomics analysis of Danggui Sini decoction on treatment of collagen-induced arthritis in rats. J Chromatogr B Analyt Technol Biomed Life Sci 2017;1061-1062:282-91.

22. Lu SH, Guan JH, Huang YL, Pan WY, Yang W, Lan H, et al. Experimental Study of Antiatherosclerosis Effects with Hederagenin in Rats. Evid Based Complement Altern Med 2015;2015:456354.

23. Gao Y, Hao J, Zhang H, Qian G, Jiang R, Hu J, et al. Protective effect of the combinations of glycyrrhizic, ferulic and cinnamic acid pretreatment on myocardial ischemia-reperfusion injury in rats. Exp Ther Med 2015;9(2):435-45.

24. Qian GQ, Ding J, Zhang X, Yin X, Gao Y, Zhao GP. Preconditioning with glycyrrhizic, ferulic, paeoniflorin, cinnamic prevents rat hearts from ischemia/reperfusion injury via endothelial nitric oxide pathway. Pharmacognosy Mag 2015;11(42):292-6.

25. Pan Y, Zhao G, Cai Z, Chen F, Xu D, Huang S, et al. Synergistic Effect of Ferulic Acid and Z-Ligustilide, Major Components of A. sinensis, on Regulating Cold-Sensing Protein TRPM8 and TPRA1 In vitro. Evid Based Complement Alternat Med 2016;2016:160247.

26. Vashistha B, Sharma A, Jain V. Ameliorative potential of ferulic acid in vincristine-induced painful neuropathy in rats: An evidence of behavioral and biochemical examination. Nutr Neurosci 2017;20(1):60-70.

27. Aswar M, Patil V. Ferulic acid ameliorates chronic constriction injury induced painful neuropathy in rats. Inflammopharmacology 2016;24(4):181-8.

28. Bennett GJ, Xie YK. A peripheral mononeuropathy in rat that produces disorders of pain sensation like those seen in man. Pain 1988;33(1):87-107.

29. Choi Y, Yoon YW, Na HS, Kim SH, Chung JM. Behavioral signs of ongoing pain and cold allodynia in a rat model of neuropathic pain. Pain 1994;59(3):369-76.

30. Kinsey SG, Long JZ, O'Neal ST, Abdullah RA, Poklis JL, Boger DL, et al. Blockade of endocannabinoid-degrading enzymes attenuates neuropathic pain. J Pharmacol Exp Ther 2009;330(3):902-10.

31. Chaplan SR, Bach FW, Pogrel JW, Chung JM, Yaksh TL. Quantitative assessment of tactile allodynia in the rat paw. J Neurosci Methods 1994;53(1):55-63.

32. Chan JK. The wonderful colors of the hematoxylin-eosin stain in diagnostic surgical pathology. Int J Surg Pathol 2014;22(1):12-32.

33. Gambella A, Porro L, Pigozzi S, Fiocca R, Grillo F, Mastracci $\mathrm{L}$, et al. Section detachment in immunohistochemistry: causes, troubleshooting, and problem-solving. Histochem Cell Biol 2017;148(1):95-101.

34. Martínez-Rojas VA, Barragán-Iglesias P, Rocha-González HI, Murbartián J, Granados-Soto V. Role of TRPV1 and ASIC3 in formalin-induced secondary allodynia and hyperalgesia. Pharmacol Rep 2014;66(6):964-71.

35. Liu M, Qiang QH, Ling Q, Yu CX, Li X, Liu S, et al. Effects of Danggui Sini decoction on neuropathic pain: experimental studies and clinical pharmacological significance of inhibiting glial activation and proinflammatory cytokines in the spinal cord. Int J Clin Pharmacol Ther 2017;55(5):453-64.

36. Peier AM, Moqrich A, Hergarden AC, Reeve AJ, Andersson 
DA, Story GM, et al. A TRP channel that senses cold stimuli and menthol. Cell 2002;108(5):705-15.

37. Pan Y, Thapa D, Baldissera L Jr, Argunhan F, Aubdool AA, Brain SD. Relevance of TRPA1 and TRPM8 channels as vascular sensors of cold in the cutaneous microvasculature. Pflugers Arch 2018;470(5):779-86.

38. Frederick J, Buck ME, Matson DJ, Cortright DN. Increased TRPA1, TRPM8, and TRPV2 expression in dorsal root ganglia by nerve injury. Biochem Biophys Res Commun 2007;358(4):1058-64.

39. Su L, Wang C, Yu YH, Ren YY, Xie KL, Wang GL. Role of TRPM8 in dorsal root ganglion in nerve injury-induced chronic pain. BMC Neurosci 2011;12:120.

40. Obata K, Katsura H, Mizushima T, Yamanaka H, Kobayashi $\mathrm{K}$, Dai $\mathrm{Y}$, et al. TRPA1 induced in sensory neurons contributes to cold hyperalgesia after inflammation and nerve. J Clin Invest 2005;115(9):2393-401.

41. Rahn EJ, Guzman-Karlsson MC, David Sweatt J. Cellular, molecular, and epigenetic mechanisms in non-associative conditioning: Implications for pain and memory. Neurobiol Learn Mem 2013;105:133-50.

42. Wei H, Karimaa M, Korjamo T, Koivisto A, Pertovaara A. Transient Receptor Potential Ankyrin 1 Ion Channel Contributes to Guarding Pain and Mechanical Hypersensitivity in a Rat Model of Postoperative Pain. Anesthesiology 2012:117(1):137-48.

43. Nazıroğlu M, Braidy N. Thermo-Sensitive TRP Channels: Novel Targets for Treating Chemotherapy-Induced Peripheral Pain. Front Physiol 2017;8:1040.

44. Amaya F, Oh-hashi K, Naruse Y, Iijima N, Ueda M, Shimosato $\mathrm{G}$, et al. Local inflammation increases vanilloid receptor 1 expression within distinct subgroups of DRG neurons. Brain Res 2003;963(1-2):190-6.

45. Hong SS, Wiley JW. Early painful diabetic neuropathy is associated with differential changes in the expression and function of vanilloid receptor 1. J Biol Chem 2005;280(1):61827.
46. Barabas ME, Stucky CL. TRPV1, but not TRPA1, in primary sensory neurons contributes to cutaneous incision-mediated hypersensitivity. Mol Pain 2013;9:9.

47. Holzer P, Holzer-Petsche U. Pharmacology of Inflammatory Pain: Local Alteration in Receptors and Mediators. Dig Dis 2009;27:24-30.

48. Horvath G, Kekesi G, Nagy E, Benedek G. The role of TRPV1 receptors in the antinociceptive effect of anandamide at spinal level. Pain 2008;134(3):277-84.

49. Ohkubo T, Shibata M, Takahashi $H$. The analgesia induced by intrathecal injection of ruthenium red. Pain 1993;54(2):21921.

50. Ding XL, Wang YH, Ning LP, Zhang Y, Ge HY, et al. Involvement of TRPV4-NO-cGMP-PKG pathways in the development of thermal hyperalgesia following chronic compression of the dorsal root ganglion in rats. Behav Brain Res 2010;208(1):194-201.

51. Ma W, Zhang Y, Bantel C, Eisenach JC. Medium and large injured dorsal root ganglion cells increase TRPV1 , accompanied by increased alpha 2C-adrenoceptor coexpression and functional inhibition by clonidine. Pain 2005;113(3):386-94.

52. Ivanusic JJ. Size, Neurochemistry, and Segmental Distribution of Sensory Neurons Innervating the Rat Tibia. J Comp Neurol 2009;517(3):276-83.

53. Hara T1, Chiba T, Abe K, Makabe A, Ikeno S, Kawakami K, et al. Effect of paclitaxel on transient receptor potential vanilloid 1 in rat dorsal root ganglion. Pain 2013;154(6):882-9.

54. Zhang Y, Wang YH, Ge HY, Arendt-Nielsen L, Wang R, Yue SW. A transient receptor potential vanilloid 4 contributes to mechanical allodynia following chronic compression of dorsal root ganglion in rats. Neurosci Lett 2008;432(3):222-7.

55. Cao DS, Yu SQ, Premkumar LS. Modulation of transient receptor potential vanilloid 4-mediated membrane currents and synaptic transmission by protein kinase C. Mol Pain 2009;5:5. 